\title{
Dexmedetomidine attenuates hypoxia/reoxygenation injury in primary neonatal rat cardiomyocytes
}

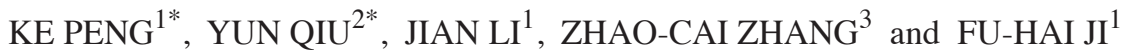 \\ ${ }^{1}$ Department of Anesthesiology, The First Affiliated Hospital of Soochow University, Suzhou, Jiangsu 215006; \\ ${ }^{2}$ Department of Anesthesiology, Suzhou Yongding Hospital, Suzhou, Jiangsu 215299; ${ }^{3}$ Department of Intensive Care Medicine, \\ The Second Affiliated Hospital of Zhejiang University School of Medicine, Hangzhou, Zhejiang 310009, P.R. China
}

Received April 3, 2016; Accepted March 23, 2017

DOI: $10.3892 /$ etm.2017.4537

\begin{abstract}
Systemic administration of dexmedetomidine provides cardioprotection against ischemia/reperfusion (I/R) injury; however, the direct effects of dexmedetomidine on cardiomyocytes have not been clarified. The present study investigated the effects of dexmedetomidine on primary neonatal rat cardiomyocytes under hypoxic/reoxygenation (H/R) conditions. In order to simulate in vivo I/R injury, primary neonatal rat cardiomyocytes were cultured under hypoxic conditions for $1 \mathrm{~h}$ and subsequently reoxygenated for $24 \mathrm{~h}$. The effects of preconditioning with dexmedetomidine $2 \mathrm{~h}$ before hypoxia and postconditioning during reoxygenation were also examined. Cellular viability and activity were analyzed by monitoring the dynamic response profile of living cells using a real-time cell analyzer system. A special scaled index, defined as the normalized cell index (NCI), was used to minimize the influence of inter-experimental variations. The dose-effect curve was generated from the area under the time-course curve values of NCI. H/R exposure markedly decreased cell viability and activity. Furthermore, no cytotoxicity was associated with a clinically relevant concentration of dexmedetomidine. Preconditioning with dexmedetomidine concentration-dependently ameliorated the reductions in $\mathrm{NCI}$ in cardiomyocytes following H/R injury. Additionally, postconditioning with dexmedetomidine improved the reductions in NCI at concentrations between 3 and 200 nM. Finally, the effect of 3-40 nM dexmedetomidine postconditioning was greater than preconditioning. These results indicated that preconditioning and postconditioning with dexmedetomidine
\end{abstract}

Correspondence to: Professor Fu-Hai Ji, Department of Anesthesiology, The First Affiliated Hospital of Soochow University, 188 Shizi Street, Suzhou, Jiangsu 215006, P.R. China

E-mail: jifuhaisuda@163.com

*Contributed equally

Key words: dexmedetomidine, hypoxia/reoxygenation, primary neonatal rat cardiomyocytes, preconditioning, postconditioning attenuated $\mathrm{H} / \mathrm{R}$ injury in primary neonatal rat cardiomyocytes at the cellular level.

\section{Introduction}

Ischemic heart disease (IHD) is a leading cause of death and disability globally (1). IN 2010, >7.0 million fatalities were caused by IHD worldwide compared with 4.5 million IHD fatalities recorded in 1980 (2). Notably, 25.6\% of IHD fatalities occurred in patients aged $<65$ years old in 2010 . Perioperatively, cardiac complications, including myocardial ischemia and infarction, are the primary causes of postoperative morbidity and mortality (3). Reperfusion, when initiated early, improves salvage of the myocardium and limits the final infarct size (4). However, reperfusion itself is capable of inducing cardiomyocyte death, known as ischemia/reperfusion (I/R) injury, for which there is still no effective therapy $(5,6)$.

Dexmedetomidine, a highly selective $\alpha-2$ adrenoceptor agonist with sedative, anxiolytic and analgesic effects, has been widely used for anesthesia and in the intensive care unit (7). Our previous studies have demonstrated that dexmedetomidine was able to reduce cardiovascular events and mortality in patients undergoing cardiac surgery $(8,9)$. Previous experiments on animals have also demonstrated benefits of dexmedetomidine when administered before ischemia (10-12); however, its use at reperfusion was reported to increase the myocardial infarct size (13). The capacity for dexmedetomidine to protect cardiac tissues against I/R injury requires further investigation.

Currently, the direct effects of dexmedetomidine on cardiomyocytes have yet to be examined. The present study was performed in order to determine the protective effects of dexmedetomidine on primary cultured neonatal rat cardiomyocytes under hypoxic/reoxygenation (H/R) conditions at the cellular level. It was hypothesized that preconditioning, but not postconditioning, with dexmedetomidine would attenuate $\mathrm{H} / \mathrm{R}$ injury in primary neonatal rat cardiomyocytes.

\section{Materials and methods}

Reagents and animals. Reagents included dexmedetomidine hydrochloride (SML0956; Sigma-Aldrich; Merck KGaA, Darmstadt, Germany), dimethyl sulfoxide (DMSO; SHBB0919V; Sigma-Aldrich; Merck KGaA), gelatin (48722; 
Sigma-Aldrich; Merck KGaA), sodium hydrosulfite $\left(\mathrm{Na}_{2} \mathrm{~S}_{2} \mathrm{O}_{4}\right.$; 80116718; Shanghai Clinical Research Center, Shanghai, China), fetal bovine serum (FBS; 16000-044), trypsin (27250-018), collagenase type II (17101-015), Hanks' balanced salt solution (14025-076; all Gibco; Thermo Fisher Scientific, Inc., Waltham, MA, USA), Dulbecco's modified Eagle medium (DMEM; SH30022.08) and phosphate-buffered saline (PBS; SH30028.01B; both HyClone; GE Healthcare Life Sciences, Logan, UT, USA). For all solutions, dexmedetomidine hydrochloride was used, as the $\mathrm{HCl}$ form has increased solubility.

A total of 80 neonatal specific-pathogen-free Sprague-Dawley rats (1-3 days old; weight, $8 \pm 2 \mathrm{~g}$; gender was not determined) were provided by the Experimental Animal Center of Soochow University (Suzhou, China). All animals were treated in accordance with the Guide for the Care and Use of Laboratory Animals published by the United States National Institute of Health (14). All rats had free access to food and water (breast-fed by mother rats) and were maintained in conditions with ventilation and a 12-h light/dark cycle. The room temperature was maintained at $22-25^{\circ} \mathrm{C}$. All experimental procedures were approved by the Ethics Committee of Experimental Animals of Soochow University.

Primary cultured neonatal rat cardiomyocyte isolation and culture. Cardiomyocytes were prepared as previously described with some modifications $(15,16)$. Rats were anesthetized by inhalation of 2-3\% ether (provided with an anesthesia apparatus; R510; RWD Industrial Co., Ltd., Shenzhen, China) and euthanized by cervical dislocation. Subsequently, the left ventricle was excised under aseptic conditions, attached tissues were removed and residual blood was washed away with PBS. The ventricle was then minced into pieces of $\sim 1 \mathrm{~mm}^{3}$ and digested in $0.07 \%$ trypsin and $0.05 \%$ collagenase II in a $37^{\circ} \mathrm{C}$ water bath for $10 \mathrm{~min}$. The cells released after the first digestion were discarded and the digestion was repeated four times. The cell supernatants from these four digestions were transferred to $10-\mathrm{ml}$ centrifuge tubes containing DMEM supplemented with $10 \%$ FBS. Cell suspensions were centrifuged for $5 \mathrm{~min}(700 \mathrm{x} \mathrm{g})$ at room temperature, filtered, and finally resuspended in DMEM supplemented with 10\% FBS at $37^{\circ} \mathrm{C}$.

To reduce contamination by fibroblasts and other cells, cardiomyocytes were purified by the differential wall adhesion method (15). Resuspended cells were plated into a T-75 culture flask (BD Biosciences, Franklin Lakes, NJ, USA) and cultured in an incubator with $5 \% \mathrm{CO}_{2}$ at $37^{\circ} \mathrm{C}$ for $90 \mathrm{~min}$. Non-adherent cells were extracted and counted with a hemocytometer (Biotechwell, Shanghai, China). Subsequently, the cells in the culture medium were transferred into 96-well gelatin-coated plates at a density of $\sim 1 \times 10^{6}$ cells $/ \mathrm{ml}$. After $96 \mathrm{~h}$ at $37^{\circ} \mathrm{C}$ $\left(5 \% \mathrm{CO}_{2}\right)$, myocyte cultures were used for subsequent experiments under $\mathrm{H} / \mathrm{R}$ conditions.

Cytotoxicity of dexmedetomidine. The plasma concentration of dexmedetomidine clinically used for sedation is $\sim 0.6 \mathrm{ng} / \mathrm{ml}$, and the maximum tolerated concentration is $10-15 \mathrm{ng} / \mathrm{ml}(16,17)$. The molecular weight of dexmedetomidine is 200.28 , therefore, $10-15 \mathrm{ng} / \mathrm{ml}$ is equivalent to 3 to $75 \mathrm{nM}$ [molar concentration $(\mathrm{mol} / \mathrm{l})=$ mass concentration $(\mathrm{g} / \mathrm{l}) /$ molecular weight].
After incubation for 40-42 h, the cultured cardiomyocytes were exposed to various concentrations of dexmedetomidine, from $10^{-9}-10^{-6} \mathrm{M}(3,10,20,40,80,200$ and $500 \mathrm{nM})$ and $10^{-6}-10^{-3} \mathrm{M}(1,3,10,30,100,300$ and $1,000 \mu \mathrm{M})$, respectively, with three replicates per group. DMSO was used as the vehicle with a concentration of $0.1 \%$ in all groups. Additionally, cell responses were continuously monitored and recorded for $72 \mathrm{~h}$.

$H / R$ injury model. An in vitro $\mathrm{H} / \mathrm{R}$ model was established, as previously described (18). $\mathrm{Na}_{2} \mathrm{~S}_{2} \mathrm{O}_{4}$ was used to generate hypoxia in the H/R injury model. $\mathrm{Na}_{2} \mathrm{~S}_{2} \mathrm{O}_{4}$ removes oxygen from the culture medium without injuring the cell membranes, and reoxygenation may be achieved by replacing the medium. Primary cultured neonatal rat cardiomyocytes were randomly divided into seven groups, with three replicates per group. Group 1 cells were cultured at $37^{\circ} \mathrm{C}$ in DMEM supplemented with 10\% FBS for $24 \mathrm{~h}$ and monitored for the duration. In groups 2-7, the culture media was replaced with serum-free DMEM and the cells were cultured with 1,2,3,4,5 and $6 \mathrm{mM}$ $\mathrm{Na}_{2} \mathrm{~S}_{2} \mathrm{O}_{4}$, respectively. These cardiomyocytes in group 2-7 were exposed to $\mathrm{Na}_{2} \mathrm{~S}_{2} \mathrm{O}_{4}$ at $37^{\circ} \mathrm{C}$ with $5 \% \mathrm{CO}_{2}$ for $1 \mathrm{~h}$, and then the culture media was replaced with DMEM with $10 \%$ FBS and the cells were monitored for $24 \mathrm{~h}$.

Preconditioning and postconditioning with dexmedetomidine. In cultured cardiomyocytes preconditioned with dexmedetomidine, cells were incubated with DMEM with 10\% FBS containing $3,10,20,40,80,200,500$ or $1,000 \mathrm{nM}$ dexmedetomidine for $2 \mathrm{~h}$ at $37^{\circ} \mathrm{C}$ before hypoxia. The culture medium was replaced with serum-free DMEM containing $\mathrm{Na}_{2} \mathrm{~S}_{2} \mathrm{O}_{4}$ for $1 \mathrm{~h}$ at $37^{\circ} \mathrm{C}$, after which $\mathrm{Na}_{2} \mathrm{~S}_{2} \mathrm{O}_{4}$ media was replaced with normal DMEM and cells were cultured for a further $24 \mathrm{~h}$ at $37^{\circ} \mathrm{C}$. In cultured cardiomyocytes postconditioned with dexmedetomidine, the reoxygenation media (DMEM supplemented with 10\% FBS) was supplemented with 3, 10, 20, 40, 80 or $200 \mathrm{nM}$ dexmedetomidine. Cells were monitored and recorded for $24 \mathrm{~h}$, and DMSO was used as the vehicle at a final concentration of $0.1 \%$ in all groups.

Real-time cell analyzer (RTCA) assay. The impedance-based RTCA system consisted of a RTCA single plate station, an RTCA computer with integrated software (version 1.1; Roche Applied Science, Mannheim, Germany), and disposable 96-well E-plates of the xCELLigence system (Cardio Plate 96; ACEA Biosciences, San Diego, CA, USA). The RTCA single plate station fits inside a standard tissue-culture incubator. The cells were seeded onto the E-plates at a density of $\sim 1 \times 10^{6} \mathrm{cells} / \mathrm{ml}$, and the electronic impedance was measured continuously to allow monitoring of cellular attachment and growth on the electrodes (19-26). In brief, the Cardio Plate 96 is a microtiter plate, which is integrated with gold microelectrodes in the bottom of each well. The impedance signal is generated by the application of low-voltage signal, creating a current between the electrodes. The interaction of cells with the electrodes impedes the current and generates the impedance signal ( $Z$ value), which is proportional to the number and the morphologic and adhesive characteristics of cells, as demonstrated in Fig. 1. Changes in rat neonatal primary cardiomyocytes were detected using methods previously described (21). 


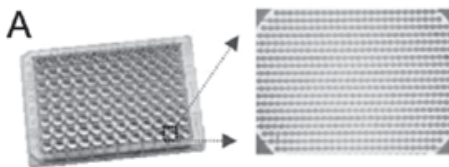

96-well E-plates

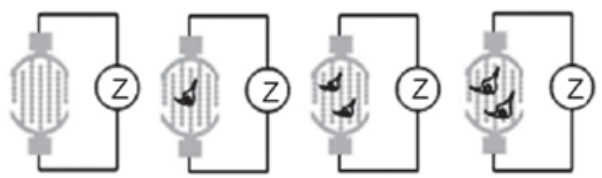

$Z$ value is proportional to the number of cells inside the well and the morphologic and adhesive characteristics
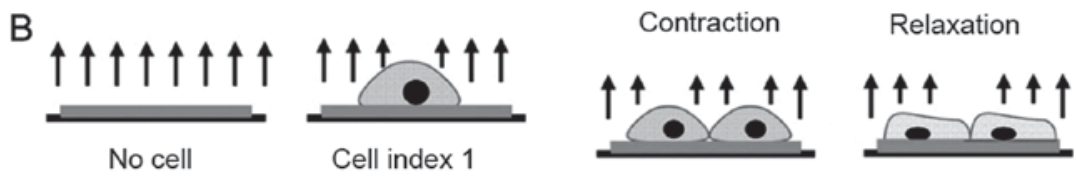

Cell index 2

Figure 1. (A) Principle of impedance readout. The impedance signal is displayed as an arbitrary unit referred to as the cell index, which is a ratio of the change in impedance to background impedance. (B) The detection of cardiomyocyte beating signal is based on the rhythmic changes of cell attachment and morphology due to contraction and relaxation, which modulates the impedance signal accordingly. Due to the fast data acquisition rate of this system, the beating of cardiomyocytes may be recorded and resolved with high temporal resolution. This figure was adapted from the study by Xi et al (21).

The RTCA system measures the impedance of cardiomyocytes and transforms the values to cell index $(\mathrm{CI})$ values to reflect the changes in beating activity. Normalized cell index (NCI) was used in the analysis to represent baseline cell activity. The NCI was calculated as the ratio of the CI value at a given time-point to the initial $\mathrm{CI}$ value at the time of exposure.

Dose-effect curve and $50 \%$ effective dose $\left(E D_{50}\right)$ calculation. The dexmedetomidine doses were transformed into logarithmic dose values and non-linear fit analysis was performed to generate dose-response curves. The model used in the present study was the following: $\mathrm{Y}=\mathrm{Bottom}+($ Top-Bottom $) /\left[1+10^{(\operatorname{LogEC} 50-\mathrm{X}) * \text { HillSlope }] \text {, where }}\right.$ the Hillslope describes the steepness of the family of curves.

An NCI of 0-100\% was used in the y-axis of the dose-effect curve. Based on the dose-response curve, the $\mathrm{ED}_{50}$ of dexmedetomidine on the NCI was calculated. Furthermore, the reliability of the $\mathrm{ED}_{50}$ calculated from a specific dose-effect curve was evaluated by the slope factor.

Statistical analysis. The area under the time-course curves (AUCs) was used to measure the cumulative effects of $\mathrm{Na}_{2} \mathrm{~S}_{2} \mathrm{O}_{4}$ and dexmedetomidine. Data were expressed as the mean \pm standard error of the mean, and one-way analysis of variance with Dunnett's post hoc test was performed using SPSS v. 19.0 statistical software (IBM SPSS, Armonk, NY, USA). P $<0.05$ was considered to indicate a statistically significant difference.

\section{Results}

Cytotoxicity of dexmedetomidine. Incubation with 3-500 nM dexmedetomidine did not significantly alter the NCI (Fig. 2A-C), indicating that dexmedetomidine was not cytotoxic to cultured cardiomyocytes when used at clinically relevant concentrations. NCI decreased significantly at dexmedetomidine concentrations $\geq 30 \mu \mathrm{M}$ compared with DMSO ( $\mathrm{P}<0.01$; Fig. 2D-F).

Effect of $\mathrm{Na}_{2} \mathrm{~S}_{2} \mathrm{O}_{4}$ on cardiomyocytes. As demonstrated in Fig. 3 , the NCI of primary cultured neonatal rat cardiomyocytes markedly decreased following exposure to $\mathrm{Na}_{2} \mathrm{~S}_{2} \mathrm{O}_{4}$. The AUCs created by all concentrations of $\mathrm{Na}_{2} \mathrm{~S}_{2} \mathrm{O}_{4}$ were significantly lower than the control $(\mathrm{P}<0.01)$ and cells incubated with a $\mathrm{Na}_{2} \mathrm{~S}_{2} \mathrm{O}_{4}$ concentration of $4 \mathrm{mM}$ gave the significantly lowest AUC compared to all other groups $(\mathrm{P}<0.01)$. As the most significant reduction in AUC for the time-course of NCI was observed in cells incubated with $4 \mathrm{mM} \mathrm{Na}_{2} \mathrm{~S}_{2} \mathrm{O}_{4}$, this concentration was selected for subsequent experiments.

Effects of dexmedetomidine on cardiomyocytes under $H / R$ injury. As demonstrated in Fig. 4A-C, preconditioning with dexmedetomidine for $2 \mathrm{~h}$ before hypoxia ameliorated reductions in NCI in primary neonatal rat cardiomyocytes exposed to $\mathrm{H} / \mathrm{R}$ injury. Dexmedetomidine significantly increased the AUC of NCI during the $24 \mathrm{~h}$ cultivation, in a concentration-dependent manner (0-10 vs. $20-40$ vs. $80-1,000 \mathrm{nM}$; all $\mathrm{P}<0.01$; Fig. 4C). The effects of different concentrations of dexmedetomidine on the NCI were calculated from the dose vs. response curve (Fig. 4D) based on the log (dose) vs. response curve (Fig. $4 \mathrm{E}$ ). The $\mathrm{ED}_{50}$ of the protective effect of dexmedetomidine on the NCI was calculated to be $40.19 \mathrm{nM}$.

Dexmedetomidine postconditioning also ameliorated reductions in NCI at all concentrations (Fig. 5A-C). The AUCs were significantly higher in the dexmedetomidine groups than in the hypoxia (DMSO) group $(\mathrm{P}<0.01)$, and the effect did not differ significantly between dexmedetomidine concentrations. The dose-effect curves are shown in Fig. 5D and E, but the exact $\mathrm{ED}_{50}$ cannot be retrieved. Additionally, 3, 10, 20, and $40 \mathrm{nM}$ dexmedetomidine postconditioning resulted in significantly higher AUCs compared with preconditioning at the same concentration (all $\mathrm{P}<0.01$; Fig. 6). All these results indicated that further studies are required to explore the effects of dexmedetomidine postconditioning on primary cultured neonatal rat cardiomyocytes.

\section{Discussion}

The present study demonstrated that preconditioning with dexmedetomidine ameliorated $\mathrm{H} / \mathrm{R}$ injury-induced reductions in $\mathrm{NCI}$ in primary cultured neonatal rat cardiomyocytes, in a concentration-dependent manner. When applied during 

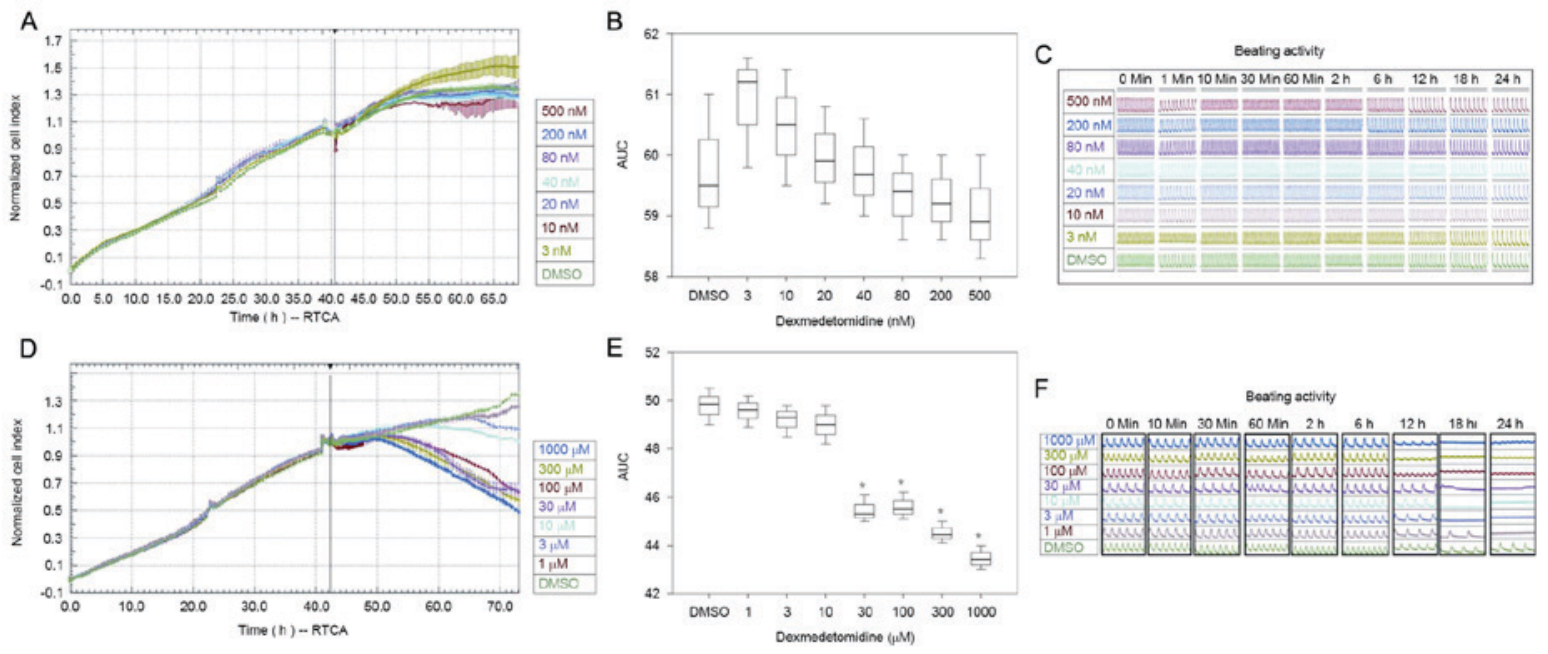

Figure 2. (A) Normalized cell index, (B) AUC calculations and (C) beating activity of neonatal rat cardiomyocytes after administration of 3-500 nM of dexmedetomidine. (D) Normalized cell index, (E) AUCs and (F) beating activity of neonatal rat cardiomyocytes after administration of 1-1,000 $\mu \mathrm{M}$ of dexmedetomidine. AUCs are presented as the mean \pm standard error of the mean. ${ }^{*} \mathrm{P}<0.01$ vs. DMSO. DMSO, dimethyl sulfoxide; AUC, area under the time-course curve; RTCA, real-time cell analyzer.
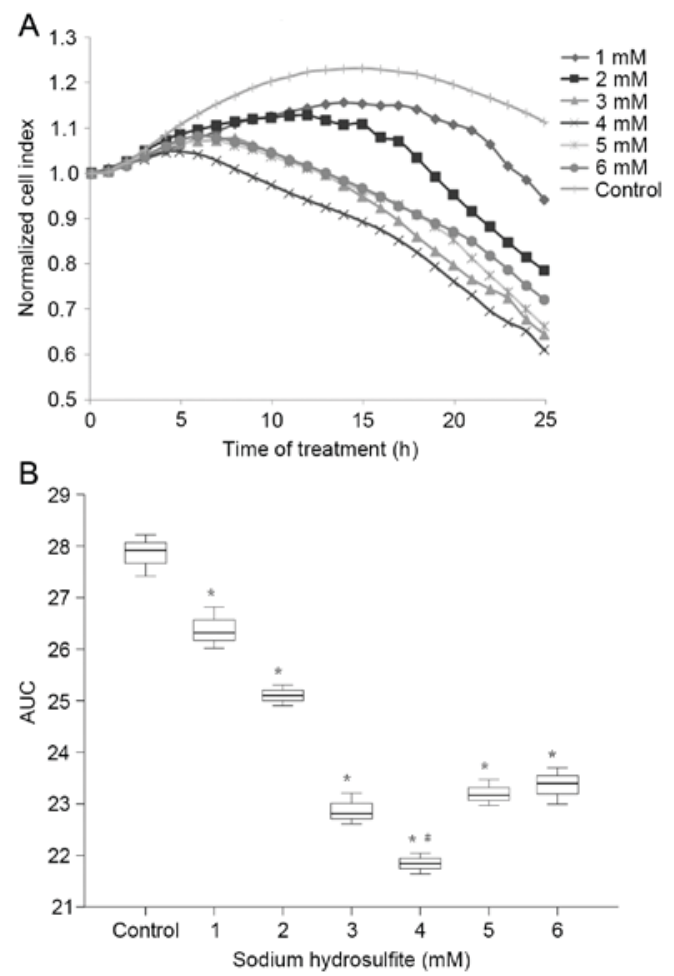

Figure 3. (A) Normalized cell index and (B) AUC calculations of neonatal rat cardiomyocytes after exposure to sodium hydrosulfite. Data are presented as the mean \pm standard error of the mean. ${ }^{*} \mathrm{P}<0.01$ vs. Control; ${ }^{~} \mathrm{P}<0.01$ vs. all other groups. Control, normal media group without sodium hydrosulfite; AUC, area under the time-course curve.

reoxygenation, however, dexmedetomidine ameliorated reductions in NCI independent of its concentration. Consistent with this, higher AUCs were observed in cells postconditioned with 3-40 $\mathrm{nM}$ dexmedetomidine than those preconditioned with equivalent dexmedetomidine concentrations.

The present study utilized rat neonatal cardiomyocytes instead of adult cells for the following reasons: The phenotype of cultured neonatal cardiomyocytes is stable, and their contractile profile during $\mathrm{H} / \mathrm{R}$ is comparable with that of in situ hearts during $\mathrm{I} / \mathrm{R}$, whereas the phenotype of isolated adult cardiomyocytes is quite different from that of in situ hearts, in part due to the loss of connections among myocytes (27). Neonatal cells form a continuous sheet, whereas adult cells are discontinuous. Furthermore, cultured neonatal rat cardiomyocytes have been demonstrated to be useful for analyzing states of oxygen- and volume-restriction, conditions that are known to stimulate anoxia and ischemia at the cellular level (28). The limitation of using neonatal cells is that they do not fully recapitulate the adult cell type, and future studies using an in vivo model are required to build on the observations of the present report.

Hypoxia was induced using a chemical oxygen scavenger, and therefore possible side effects of $\mathrm{Na}_{2} \mathrm{~S}_{2} \mathrm{O}_{4}$ need to be considered. In previous studies, hydrosulfite was demonstrated to not be equivalent to authentic hypoxia because of little similarity to hypoxic vasoconstriction $(29,30)$. Although hypoxic ventilation and hydrosulfite both lower $\mathrm{PO}_{2}$, only hydrosulfite generates activated oxygen species, increases lung weight, and impairs vascular responsiveness to vasoconstrictors $(29,30)$. According to previous research, $\mathrm{Na}_{2} \mathrm{~S}_{2} \mathrm{O}_{4}$ may still be a useful agent for inducing hypoxia in various types of cell models, including primary neonatal rat cardiomyocytes $(18,31-33)$.

The RTCA is a novel technique for continuous monitoring of cell adherence, proliferation, migration and cytotoxicity (21). This technology allows for uninterrupted, label free, real-time analysis of the cells over the entire course of the experiment, which provides a dynamic and sensitive assessment beyond the scope of single time-point assays (22). A number of studies have demonstrated that this system is a valid method for analyzing the effects of compound cytotoxicity, and measuring cell death of various cell types, including primary cultured neonatal rat cardiomyocytes (19-26).

The advantages of the RTCA technology include its capacity for noninvasive measurements that do not require cellular labeling or overexpression of reporter proteins, and no or minimal intervention in cell physiology. Additionally, 

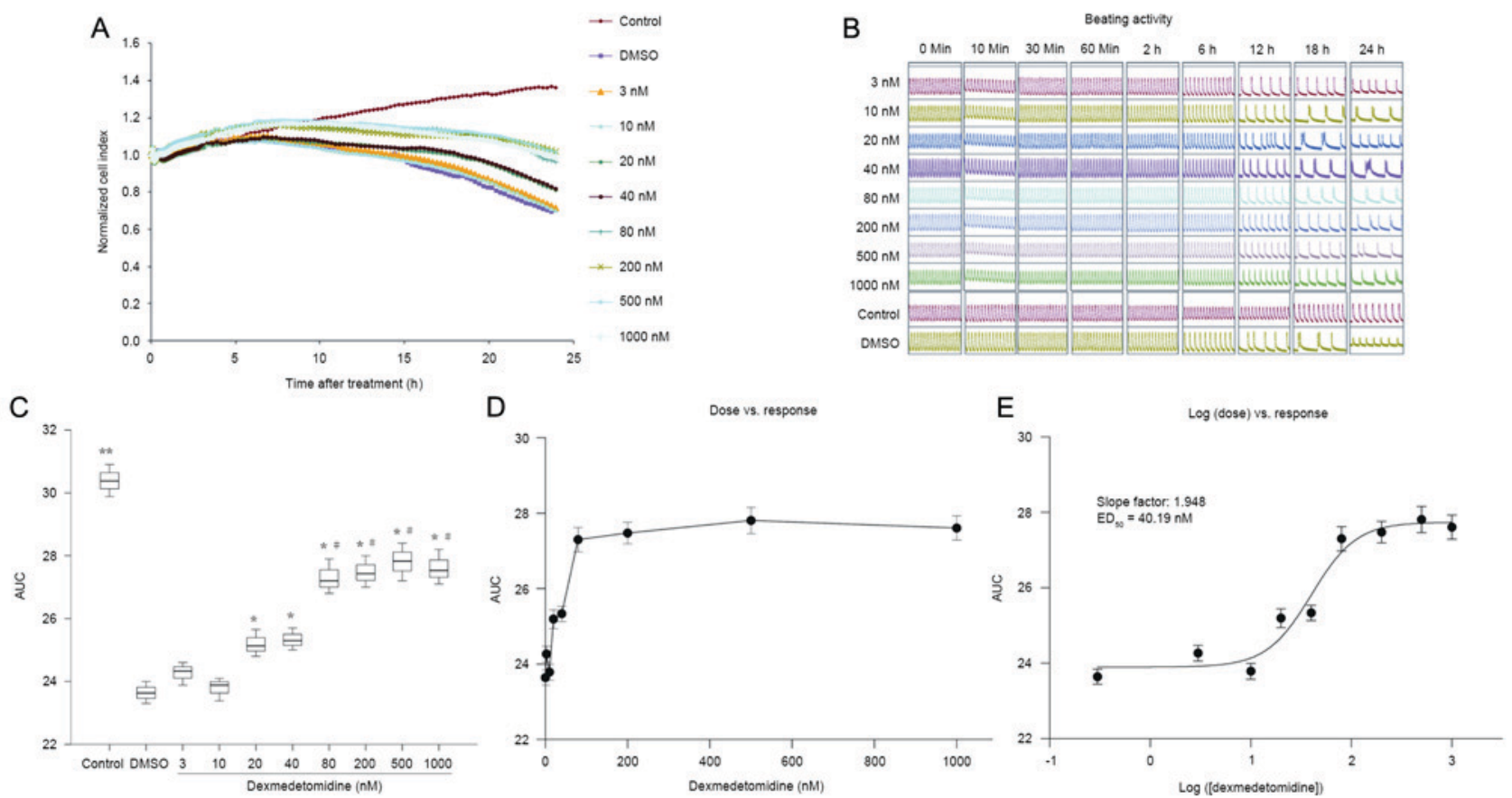

Figure 4. (A) Normalized cell index and (B) beating activity of primary cultured neonatal rat cardiomyocytes treated with dexmedetomidine $2 \mathrm{~h}$ before hypoxia (preconditioning). (C) AUC graphs for different groups were calculated to perform statistical analysis. (D) Dose-effect and (E) log (dose) -effect curves. Data are presented as the mean \pm standard error of the mean. ${ }^{*} \mathrm{P}<0.01$ vs. DMSO group $(0 \mathrm{nM}$ dexmedetomidine); $\mathrm{P}<0.01$ vs. 20 and $40 \mathrm{nM}$ dexmedetomidine group; ${ }^{* *} \mathrm{P}<0.01$ vs. all other groups. Control, normal media group without $\mathrm{Na}_{2} \mathrm{~S}_{2} \mathrm{O}_{4} ; \mathrm{DMSO}$, dimethyl sulfoxide; $\mathrm{AUC}$, area under the time-course curve; $\mathrm{ED}_{50}$, $50 \%$ effective dose.
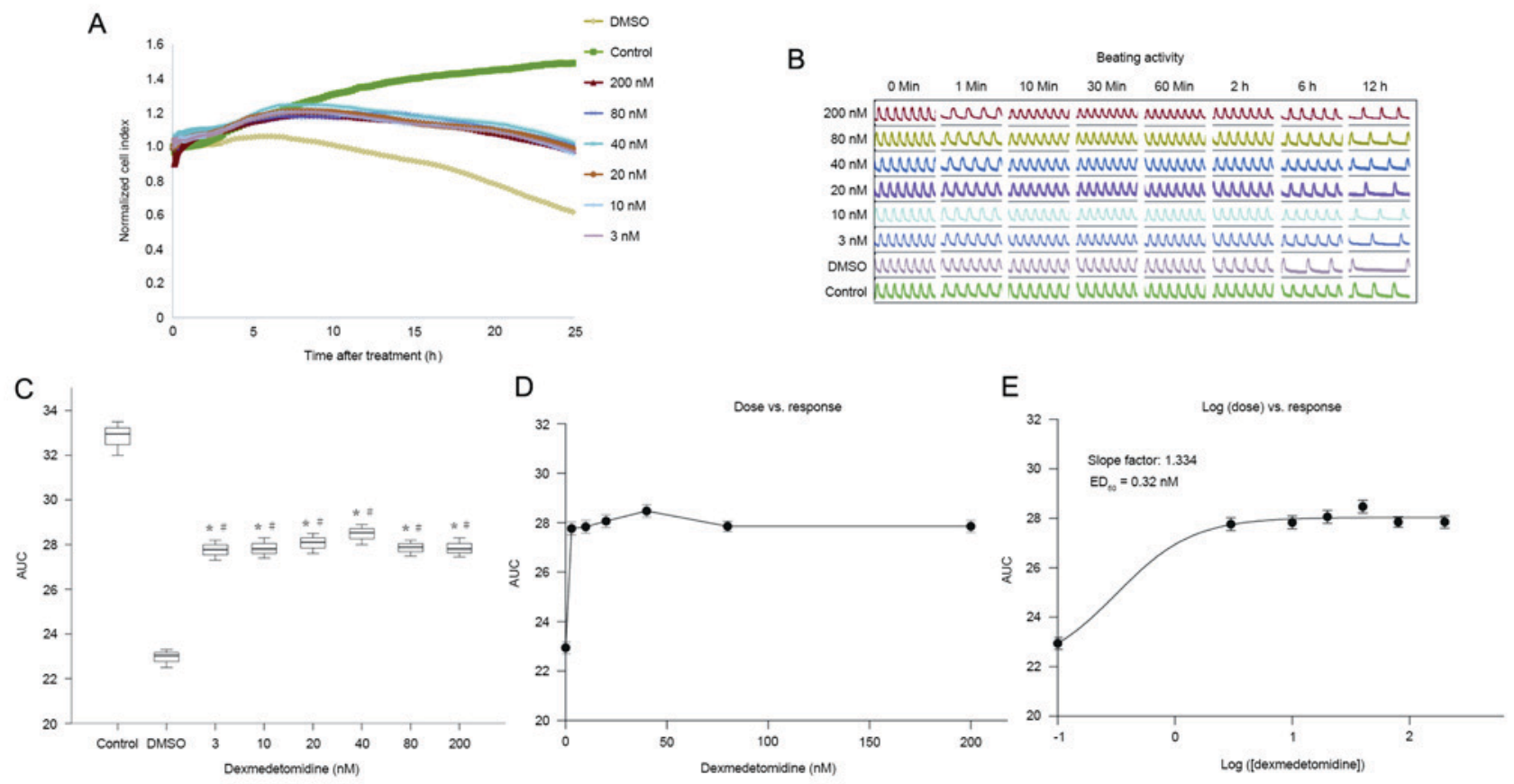

Figure 5. (A) Normalized cell index and (B) beating activity of primary cultured neonatal rat cardiomyocytes treated with dexmedetomidine at reoxygenation (postconditioning). (C) AUC graphs for different groups were calculated to perform statistical analysis. (D) Dose-effect and (E) log (dose)-effect curves. Data are presented as the mean \pm standard error of the mean. ${ }^{*} \mathrm{P}<0.01$ vs. control; ${ }^{*} \mathrm{P}<0.01 \mathrm{vs}$. DMSO $(0 \mathrm{nM}$ dexmedetomidine $)$. Control, normal media group without $\mathrm{Na}_{2} \mathrm{~S}_{2} \mathrm{O}_{4}$; DMSO, dimethyl sulfoxide; AUC, area under the time-course curve; $\mathrm{ED}_{50}, 50 \%$ effective dose.

its real-time sensitive cell-monitoring platform allows for real-time kinetic evaluation and is easy to set up. The RTCA technology may be used for pharmacology and toxicology studies with a reasonable throughput $(22,24)$, and, therefore, represents a powerful and reliable tool for drug discovery (34).

Generally, the cytotoxicity of the experimental drug is evaluated prior to analyzing its effect on cell physiology. Thus, 


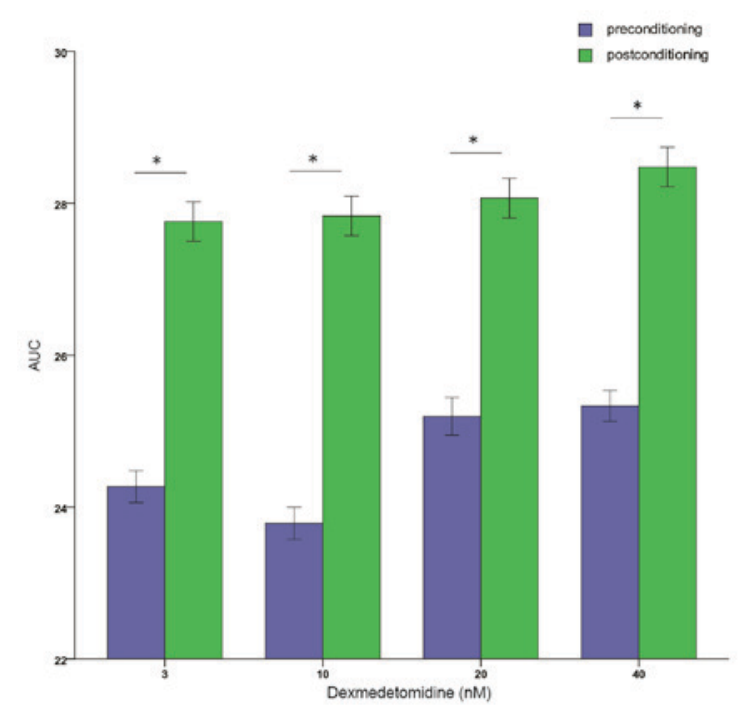

Figure 6. AUC calculations of neonatal rat cardiomyocytes after preconditioning and postconditioning administration of 3-40 $\mathrm{nM}$ of dexmedetomidine, respectively. Data are presented as the mean \pm standard error of the mean ${ }^{*} \mathrm{P}<0.01$. AUC, area under the time-course curve.

the cytotoxicity of dexmedetomidine was determined before investigating its capacity to protect cardiomyocytes following $\mathrm{H} / \mathrm{R}$ injury. In the present study, a clinically relevant concentration range of dexmedetomidine was observed to not have significant adverse effects on cardiomyocyte viability.

Previous studies have reported that systemic administration of $\alpha-2$ adrenergic agonists, clonidine or dexmedetomidine, were able to provide myocardial protection in vivo by attenuating the catecholamine response to ischemic stress (35-37) and improving the myocardial oxygen balance $(38,39)$. Despite these benefits, the direct effects of $\alpha-2$ adrenergic agonists on the coronary vasculature are controversial. Some studies have indicated that $\alpha-2$ adrenergic coronary vasoconstriction exerts favorable effects on the ischemic myocardium, preventing transmural redistribution of blood flow away from the endocardium and improving the subendocardial to subepicardial blood flow ratio $(40,41)$. Other studies have demonstrated that the activation of coronary vascular $\alpha-2$ adrenergic receptors induced post-stenotic coronary vasoconstriction and myocardial ischemia (42). However, the direct effects of dexmedetomidine on cardiomyocytes have not been determined.

To the best of our knowledge, the present study is the first to demonstrate the protective effects of dexmedetomidine on $\mathrm{H} / \mathrm{R}$ injury in a neonatal rat cardiomyocyte model. As expected, preconditioning with dexmedetomidine significantly ameliorated the loss of viability resulting from $H / R$ injury in a concentration-dependent manner, with no observed cardiomyocyte toxicity. The in vitro $\mathrm{H} / \mathrm{R}$ model used in the present study excluded local in vivo cardiac and central sympathetic effects, thus revealing the direct and specific effects of dexmedetomidine on cardiomyocytes. Furthermore, the present study simulated plasma concentrations of dexmedetomidine that are frequently used clinically for sedation, and it was demonstrated that the cell number and activity was better preserved by higher concentrations of dexmedetomidine. This observation suggested that dexmedetomidine preconditioning with higher concentrations may achieve greater protection against H/R injury of cardiomyocytes.

Cardioprotective therapy following reperfusion is more clinically feasible than preconditioning, as the onset of reperfusion is predictable and is under the control of the clinicians. However, previous studies have reported that administration of dexmedetomidine after reperfusion did not exert a direct protective effect on left ventricular dysfunction, and even increased myocardial infarct size in isolated rat hearts $(13,43)$. Contrary to our secondary hypothesis, the results of the present study indicated that postconditioning with dexmedetomidine was also able to attenuate $\mathrm{H} / \mathrm{R}$ injury of cardiomyocytes. Notably, dexmedetomidine applied shortly after the onset of reoxygenation improved cell survival even more effectively than when applied before hypoxia. There are two possible explanations for this observation: i) Preconditioning was applied $2 \mathrm{~h}$ before hypoxia, whereas postconditioning was initiated at the time of reoxygenation and therefore, the timing of dexmedetomidine administration may have caused different dose-effects on the cardiomyocytes; and ii) the culture medium containing dexmedetomidine was replaced with media containing $\mathrm{Na}_{2} \mathrm{~S}_{2} \mathrm{O}_{4}$ during preconditioning, which may affect the protective effects of dexmedetomidine on the cultured cardiomyocytes. As the predominant conclusion of the present study is that dexmedetomidine attenuates H/R injury under both preconditioning and postconditioning, a future direction from here is to determine the lowest efficacious concentration.

The conclusions of the present study are limited by its scope. Firstly, an in vitro experimental H/R model was used to simulate I/R injury, and condition-dependent differences may exist between models. Secondly, rat neonatal cardiomyocytes were used instead of adult cardiomyocytes, and the physiological benefits of dexmedetomidine will need to be investigated using adult rat or human cells to more accurately reflect the clinical application of dexmedetomidine. Furthermore, investigation into the precise mechanisms responsible for the cardioprotective effects of dexmedetomidine is now in progress. In summary, the present study is a preliminary investigation that was performed to determine the effects of preconditioning and postconditioning with dexmedetomidine on cardiomyocyte protection against $\mathrm{H} / \mathrm{R}$ injury at the cellular level, as measured by improved cell physiology assays.

\section{Acknowledgements}

The present study was supportedby the National Natural Science Foundation of China (grant nos. 81471835 and 81471889) and the Science and Technology Development Projects of Soochow (grant no. SYSD2013073). The present work was a follow-up study of the abstract presented at the Anesthesiology Annual Meeting of the American Society of Anesthesiologists Oct 27th 2015 in San Diego, CA, USA as abstract no. A4171.

\section{References}

1. Sluijter JP, Condorelli G, Davidson SM, Engel FB, Ferdinandy P, Hausenloy DJ, Lecour S, Madonna R, Ovize M, Ruiz-Meana M, et al: Novel therapeutic strategies for cardioprotection. Pharmacol Ther 144: 60-70, 2014. 
2. Moran AE, Forouzanfar MH, Roth GA, Mensah GA, Ezzati M, Murray CJ and Naghavi M: Temporal trends in ischemic heart disease mortality in 21 world regions, 1980 to 2010: The Global Burden of Disease 2010 study. Circulation 129: 1483-1492, 2014

3. Poldermans D, Hoeks SE and Feringa HH: Pre-operative risk assessment and risk reduction before surgery. J Am Coll Cardiol 51: 1913-1924, 2008.

4. Keeley EC, Boura JA and Grines CL: Primary angioplasty versus intravenous thrombolytic therapy for acute myocardial infarction: A quantitative review of 23 randomised trials. Lancet 361 13-20, 2003.

5. Kloner RA: Does reperfusion injury exist in humans? J Am Coll Cardiol 21: 537-545, 1993.

6. Hausenloy DJ and Yellon DM: Myocardial ischemia-reperfusion injury: A neglected therapeutic target. J Clin Invest 123: 92-100, 2013.

7. Gerlach AT, Murphy CV and Dasta JF: An updated focused review of dexmedetomidine in adults. Ann Pharmacother 43 2064-2074, 2009.

8. Ji F, Li Z, Nguyen H, Young N, Shi P, Fleming N and Liu H: Perioperative dexmedetomidine improves outcomes of cardiac surgery. Circulation 127: 1576-1584, 2013.

9. Ji F, Li Z, Young N, Moore P and Liu H: Perioperative dexmedetomidine improves mortality in patients undergoing coronary artery bypass surgery. J Cardiothorac Vasc Anesth 28: 267-273, 2014.

10. Yoshitomi O, Cho S, Hara T, Shibata I, Maekawa T, Ureshino H and Sumikawa K: Direct protective effects of dexmedetomidine against myocardial ischemia- reperfusion injury in anesthetized pigs. Shock 38: 92-97, 2012.

11. Okada H, Kurita T, Mochizuki T, Morita K and Sato S: The cardioprotective effect of dexmedetomidine on global ischaemia in isolated rat hearts. Resuscitation 74: 538-545, 2007.

12. Ibacache M, Sanchez G, Pedrozo Z, Galvez F, Humeres C, Echevarria G, Duaso J, Hassi M, Garcia L, Díaz-Araya G and Lavandero S: Dexmedetomidine preconditioning activates pro-survival kinases and attenuates regional ischemia/reperfusion injury in rat heart. Biochim Biophys Acta 1822: 537-545, 2012.

13. Mimuro S, Katoh T, Suzuki A, Yu S, Adachi YU, Uraoka M, Sano $\mathrm{H}$ and Sato $\mathrm{S}$ : Deterioration of myocardial injury due to dexmedetomidine administration after myocardial ischaemia Resuscitation 81: 1714-1717, 2010.

14. Guide for the Care and Use of Laboratory Animals. NIH Publication No. 85-23, 1996.

15. Zhang C, Lin G, Wan W, Li X, Zeng B, Yang B and Huang C: Resveratrol, a polyphenol phytoalexin, protects cardiomyocytes against anoxia/reoxygenation injury via the TLR4/NF- $\mathrm{KB}$ signalling pathway. Int J Mol Med 29: 557-563, 2012.

16. Chrysostomou C and Schmitt CG: Dexmedetomidine: Sedation, analgesia and beyond. Expert Opin Drug Metab Toxicol 4 619-627, 2008

17. Ebert TJ, Hall JE, Barney JA, Uhrich TD and Colinco MD: The effects of increasing plasma concentrations of dexmedetomidine in humans. Anesthesiology 93: 382-394, 2000

18. Ren C, Bao YR, Meng XS, Diao YP and Kang TG: Comparison of the protective effects of ferulic acid and its drug-containing plasma on primary cultured neonatal rat cardiomyocytes with hypoxia/reoxygenation injury. Pharmacogn Mag 9: 202-209, 2013.

19. Garcia SN, Gutierrez L and McNulty A: Real-time cellular analysis as a novel approach for in vitro cytotoxicity testing of medical device extracts. J Biomed Mater Res Part A 101: 2097-2106, 2013

20. Pan T, Khare S, Ackah F, Huang B, Zhang W, Gabos S, Jin C and Stampfl M: In vitro cytotoxicity assessment based on $\mathrm{KC}(50)$ with real-time cell analyzer (RTCA) assay. Comput Bio Chem 47: 113-120, 2013.

21. Xi B, Wang T, Li N, Ouyang W, Zhang W, Wu J, Xu X, Wang X and Abassi YA: Functional cardiotoxicity profiling and screening using the xCELLigence RTCA cardio system. J Lab Autom 16: 415-421, 2011.

22. Atienzar FA, Tilmant K, Gerets HH, Toussaint G, Speeckaert S, Hanon E, Depelchin O and Dhalluin S: The use of real-time cell analyzer technology in drug discovery: Defining optimal cell culture conditions and assay reproducibility with different adherent cellular models. J Biomol Screen 16: 575-587, 2011.
23. Kirstein SL, Atienza JM, Xi B, Zhu J, Yu N, Wang X, Xu X and Abassi YA: Live cell quality control and utility of real-time cell electronic sensing for assay development. Assay Drug Dev Technol 4: 545-553, 2006.

24. Quereda JJ, Martínez-Alarcón L, Mendoça L, Majado MJ, Herrero-Medrano JM, Pallarés FJ, Ríos A, Ramírez P, Muñoz A and Ramis G: Validation of xCELLigence real-time cell analyzer to assess compatibility in xenotransplantation with pig-to-baboon model. Transplant Proc 42: 3239-3243, 2010.

25. Huang L, Xie L, Boyd JM and Li XF: Cell-electronic sensing of particle-induced cellular responses. Analyst 133: 643-648, 2008

26. Xing JZ, Zhu L, Jackson JA, Gabos S, Sun XJ, Wang XB and $\mathrm{Xu} \mathrm{X}$ : Dynamic monitoring of cytotoxicity on microelectronic sensors. Chem Res Toxicol 18: 154-161, 2005.

27. Yamashita N, Nishida M, Hoshida S, Kuzuya T, Hori M, Taniguchi N, Kamada T and Tada M: Induction of manganese superoxide dismutase in rat cardiac myocytes increases tolerance to hypoxia $24 \mathrm{~h}$ after preconditioning. J Clin Invest 94 2193-2199, 1994

28. Iwaki K, Chi SH, Dillmann WH and Mestril R: Induction of HSP70 in cultured rat neonatal cardiomyocytes by hypoxia and metabolic stress. Circ Res 87: 2023-2032, 1993.

29. Archer SL, Hampl V, Nelson DP, Sidney E, Peterson DA and Weir EK: Dithionite increases radical formation and decreases vasoconstriction in the lung. Evidence that dithionite does not mimic alveolar hypoxia. Circ Res 77: 174-181, 1995.

30. Yu MF, Gorenne I, Su X, Moreland RS and Kotlikoff MI: Sodium hydrosulfite contractions of smooth muscle are calcium and myosin phosphorylation independent. Am J Physiol 275: L976-L982, 1998.

31. Sun J, Li YZ, Ding YH, Wang J, Geng J, Yang H, Ren J, Tang JY and Gao J: Neuroprotective effects of gallic acid against hypoxia/reoxygenation-induced mitochondrial dysfunctions in vitro and cerebral ischemia/reperfusion injury in vivo. Brain Res 1589: 126-139, 2014.

32. Luo L, Lü L, Lu Y, Zhang L, Li B, Guo K, Chen L, Wang Y, Shao Y and Xu J: Effects of hypoxia on progranulin expression in HT22 mouse hippocampal cells. Mol Med Rep 9: 1675-1680, 2014.

33. Rigatto H, Fitzgerald SF, Willis MA and Yu C: In search of the real respiratory neurons: Culture of medullary fetal cells sensitive to CO2 and low pH. Biol Neonate 65: 149-155, 1994.

34. Abassi YA, Xi B, Zhang W, Ye P, Kirstein SL, Gaylord MR, Feinstein SC, Wang X and Xu X: Kinetic cell-based morphological screening: Prediction of mechanism of compound action and off-target effects. Chem Biol 16: 712-723, 2009.

35. Willigers HM, Prinzen FW, Roekaerts PM, de Lange S and Durieux ME: Dexmedetomidine decreases perioperative myocardial lactate release in dogs. Anesth Analg 96: 657-664, 2003.

36. Meissner A, Weber TP, Van Aken H, Zbieranek K and Rolf $\mathrm{N}$ : Clonidine improve recovery from myocardial stunning in conscious chronically instrumented dogs. Anesth Analg 87: 1009-1014, 1998

37. Roekaerts PM, Prinzen FW and De Lange S: Beneficial effects of dexmedetomidine on ischaemic myocardium of anaesthetized dogs. Br J Anaesth 77: 427-429, 1996.

38. Lawrence CJ, Prinzen FW and de Lange S: Hemodynamic and coronary vascular effects of dexmedetomidine in the anesthetized goat. Acta Anaesthesiol Scand 41: 830-836, 1997.

39. Jalonen J, Halkola L, Kuttila K, Perttilä J, Rajalin A, Savunen T, Scheinin $\mathrm{M}$ and Valtonen $\mathrm{M}$ : Effects of dexmedetomidine on coronary hemodynamics and myocardial oxygen balance. J Cardiothorac Vasc Anesth 9: 519-524, 1995.

40. Miyamoto MI, Rockman HA, Guth BD, Heusch G and Ross J Jr: Effect of alpha-adrenergic stimulation on regional contractile function and myocardial blood flow with and without ischemia. Circulation 84: 1715-1724, 1991.

41. Chilian WM and Ackell PH: Transmural differences in sympathetic coronary constriction during exercise in the presence of coronary stenosis. Circ Res 62: 216-225, 1988.

42. Heusch G, Schipke J and Thämer V: Clonidine prevents the sympathetic initiation and aggravation of poststenotic myocardial ischemia. J Cardiovasc Pharmacol 7: 1176-1182, 1985.

43. Guo H, Takahashi S, Cho S, Hara T, Tomiyasu S and Sumikawa K: The effects of dexmedetomidine on left ventricular function during hypoxia and reoxygenation in isolated rat hearts. Anesth Analg 100: 629-635, 2005. 